\title{
PoP AS PROCESS: \\ The Digitalization of Groove, Form and Time
}

\author{
Feature Article
}

ANDERS REUTER

INDEPENDENT RESEARCHER

\begin{abstract}
Pop music production is increasingly dominated by practices from hip-hop and EDM. The shift is hosted by a new generation of the digital audio workstation (DAW) that not only connects with but also reflects contemporary ubiquitous computing culture. This article examines the temporal consequences of this digitalization of pop production and how it challenges traditional interpretations of the relationship between groove, repetition and teleology. Using analysis of three current pop tracks, digital culture theory and process philosophy I approach contemporary pop temporality through a process paradigm. I argue that groove is rooted in signification of technology and genre-based practices rather than performance-based notions of difference, tension or timing discrepancy. Pop production is based on a hybridization of human and algorithmic agency which entails new kinds of temporal ambiguity, multiplicity, precision and infinitesimal detail. In conclusion, I discuss how these novel temporal traits manifest themselves prereflexively as tacit knowledge.
\end{abstract}

KEYWORDS: pop; music production; groove; digitalization; process philosophy

Anders Reuter received his $\mathrm{PhD}$ from University of Copenhagen in 2021. Entitled "Pop Processing: The Digitalization of Musical Time and Space", it intersected popular music studies and new media studies. He also works as a journalist, writer and music producer.

Dancecult: Journal of Electronic Dance Music Culture 13(1): 2-21 ISSN 1947-5403 @2021 Dancecult http://dj.dancecult.net https://dx.doi.org/10.12801/1947-5403.2021.13.01.01 


\section{INTRODUCTION}

"Time has changed in the wake of the digital computational revolution" (Hansen 2009: 295). New media theorist Mark Hansen even calls it a banal proposition because of the "extensive work of temporalizing that is currently carried out by technical artifacts in our world today" (2009: 298). Indeed, music holds a particular relationship to time. As philosopher Susanne Langer writes: "music makes time audible, and its form and continuity sensible" (1953: 110). Perhaps more than any musical form, pop is inherently entangled with the computational shift from inception to distribution to reception. Concurrently, hip-hop and EDM and their temporalities have in the past decades moved and adapted to the pop single format. This assimilation has arguably found a new home or even realisation in recent iterations of the digital audio workstation (DAW), offering new production practices in pop music production. ${ }^{1}$ The question then arises: What does the computational shift mean for music's relationship to time? Or, more specifically, how does digitalization affect essential temporal features of pop music such as groove, form and teleology?

I begin this article with a critical discussion of the performance-paradigm in popular music theory and hold it against hip-hop and EDM-theory on the relationship between repetition and technology. I then analyse how hip-hop and EDM-derived characteristics gain particular digital or even algorithmic character in contemporary pop music production in three tracks: Martin Garrix \& Dua Lipa’s "Scared to Be Lonely", Post Malone’s "rockstar" and Katy Perry's "Chained to the Rhythm". They present various, and often dissimilar, kinds of temporal discrepancies and teleologies which, I argue, are part of a shift towards a musical process ontology. I turn to digital media theory to discuss the musical implications of this computational temporality in pop music. Ubicomp (ubiquitous computing) culture displays new heterogenous temporal properties that can be traced in digital pop music's novel levels of automation, precision, synchronicity and multiplicity that fundamentally defy human scale. I conclude that music production's increasing shift towards a digital process ontology challenges anthropocentric, performance and difference-based interpretations of agency and time. Instead, rhythm and time in pop are better understood as digital processes that signify particular music technologies and their genre-based practices. With inspiration from process philosophy, I discuss how a focus on how sounds multiply, relate or even grasp each other across loops and signal processing could present a new path for musical analysis. In the end, I argue that-in contrast to musicology's dominant phenomenological interpretations-these processes manifest prereflexively, as tacit knowledge of the technology-bound processes unfolding.

\section{Repetition, Difference and Processual Technology Signification}

A key question in popular music studies is the relationship between rhythm and time. In theory on performed popular music rhythm, the combination of repetition and spontaneous irregularity is understood as integral in music's fundamental temporal push or drive forward. This is probably best described in Charles Keil's influential work on rhythm, 
in which he describes what he calls participatory discrepancies. According to Keil, music is a communal or social activity and for it to be "personally involving and socially valuable, [it] must be 'out of time' and 'out of tune'" (1987: 275). For Keil, these discrepancies are corporeally bound and create what he calls the music's vital drive. Similar thoughts can be found in Anne Danielsen's research into groove and time. ${ }^{2}$ Here, drive is created via microrhythmic tension or through structural tension, which is, for example, the tension created by counter-rhythms such as the $4: 3$ pattern. According to Danielsen, a groove is heard as repetition with a difference or sameness depending on the listeners' resolution and experience; "a low-resolution, non-confident listener will probably tend to hear the 'same thing,' despite considerable differences from one repetition to the next" (Danielsen 2018: $40-41)$. In other words, from this phenomenological perspective, it is the microrhythmic or structural tension that affords (the confident) listener the feeling of groove, of time and the work moving forward. Nevertheless, to Danielsen, it is not decisive whether a groove is exact or not, because even in "music performed by machines and not human beings-every repetition may be experienced as repetition with a difference, because the time is different" (Danielsen 2018: 49). ${ }^{3}$

Mark Butler's EDM-research switches the perspective from human performance to technology. To him, EDM sidesteps the timing variations that tend to inform human performance. "In the ways in which they repeat, EDM compositions actively model technology", and this turns exact repetition into "an intentionally cultivated creative strategy" (Butler 2014: 188). Instead of negating the existence of repetition without difference, exact repetition can be valued for what it does, making it "possible to appreciate its epistemological reality" (Butler 2014: 190). Yet the discrepancies of, for example, hiphop grooves do present a type of tension, or perhaps rather a particular negotiation of tension. Mike D'Errico writes about the relationship between repetition and discrepancy: "when a rhythmically dissonant moment is repeated, what was previously a rupture in the coherence of the groove becomes transitional material, enhancing the effect of the anacrusis and solidifying the stability of the beat" (D'Errico 2015: 287). The consequence of D'Errico's argument is that instability, rupture and discrepancies within the loop can actually reinforce stability. In fact, the amount of detail being repeated verbatim emphasizes its inhuman automated perfection. Instead of understanding discrepancies as signifiers of human performance, this perspective allows us to see temporal signification as intricately bound to technology.

Accordingly, various types of electronic grooves will have specific origins in practices that are equally historical, genre-related and directly embedded in specific types of music technology. As Joseph Schloss writes on hip-hop rhythm: "The circuitry and programming of different models of samplers are believed to impart special characteristics to the music (perhaps the best known of these characteristics is the legendary 'MPC swing', a rhythmic idiosyncrasy first noted in the Akai MPC 60 sampler, circa 1988)" (Schloss 2014: 202). Particular music technologies afford particular rhythms. From turntable-cuts between breakbeats on vinyl to drum machines to samplers and MPC's, hip-hop aesthetics is based on a variety of technology-bound grooves. 
The technological frames-the instruments shaping hip-hop as well as EDM's loopshave many (inter)faces, each with their own temporalities, and today these are all available in the grid of the contemporary DAW. Here, the affordances of previous music technologiespossibilities as well as limitations-are transposed to a digital context defined by a novel level and type of processual fitting, juxtaposition and interconnection. Instead of blunt technological determinism, it is based on complex layers of technological signification. However, while the DAW can do and signify virtually anything in terms of groove and time, the more recent iterations of the DAW also afford specific and novel temporal features of their own.

\section{NeW Temporalities in the DAW}

The first generation of 1990s DAWs was based on a logic from the analogue recording studio. ${ }^{4}$ Industry leading programs such as Pro Tools, Cubase and Logic had interfaces that were built on recording, organising and editing sound and MIDI through skeuomorphic representations of the recording studio. This included virtual effect racks and mixing desks and a tape-recorder logic of start, stop and record. The recording timeline was horizontal, an axis of time upon which sound events could be stacked vertically. Steve Goodman writes: "As with European musical notation's inheritance from written text, digital audio software sequencers have inherited the habit of left-to-right visual scanning. The timeline constitutes the spatialization of the clock into a horizontal time" (Goodman 2008: 257). The timeline of the first generation of the DAWs thus seemingly represents a traditional linear temporality. Yet, as Goodman argues, the temporal homogeneity begins to dissolve in the operations of the interface. "A temporal sequence of sounds suddenly occupies an area of the computer screen. What is opened up by this spatialization is the ease of temporal recombination" (Goodman 2008: 257). He concludes that this facilitates nonlinear digital editing, which is different from analogue fast-forwarding and rewinding.

However, recent developments in digital music production extend beyond temporal juxtapositions of cut-and-paste techniques and manipulating time by, for example, timestretching (changing the speed without changing pitch) or pitch scaling (changing pitch without changing speed). The dissolution of temporal linearity has been taken a step further in a new generation of highly popular DAWs such as FL Studio and Ableton Live. These put the multiplicity, grid-based sequencing of interrelational parts and algorithmic processing at the centre of music production. FL Studio can essentially perform the same functions as traditional DAWs, and its layout is in many ways similar to hardware-sequencers like the Roland TR-808 or the TB-303. However, it is as software innately based on fast, intuitive and beat-centred sequencing in the interface. While the time-axis-bound regime of the timecursor does to some extent prevail in its sequencer, it does so almost multidimensionally, across windows of loops within loops, audio and MIDI metadata. A new automatised relationship between detail and the whole is therefore created; singular edits potentially apply to functions across fragments and the whole track. It is a practice based on a fast, programmable multiplicity where temporal events are constantly interconnected. 
Ableton Live represents a perhaps even more conspicuous alternative to the traditional timeline. It consists of two view types and concurrent work modes: Arrangement View and Session View. The latter offers an inherently loop-based operationality. Anything from short sound bites to whole albums can be triggered, manipulated and interrelated on the fly via a grid of coloured squares. As its name implies, it is a musical practice that is based on a live and improvisational approach to producing music. While Session View in many ways works as a virtualisation of the logic of an MPC, its mouldable interface control and connectivity of processes differs. Music production becomes processual-something ongoing, running. Accordingly, in a pop production context, the final product (the track) becomes a screen-dump, as all the running processes are bounced down, frozen and committed to a definitive format.

Additionally, the use of samples and prepacked loops has been taken to a new level in cloud-based music production. When producers across the globe can access and use the same downloadable presets, samples and full loops (for instance through subscriptions to services such as Splice, Noiiz or Loopcloud) the creative emphasis is put on their use and relation to other parts. This entails an increased emphasis on processual interconnectivity.

In a sense, this is a practice or logic that is already embedded in the modularity of sound synthesis. Essentially, analogue sound synthesis is based on fluctuating voltage used to shape and control other kinds of fluctuating voltages. It is a system of signal processing through the interconnected modelling of sound across parameters. From this perspective, music production becomes a macro-synthesis of sound working as a hybrid algorithmic structure. It is not just in its basis on loops, but in the conjunction of multiple automated functions that process and define each other mutually.

\section{From the Dancefloor to the Pop Charts}

This shift in practices in music production has happened almost concurrently with pop music's assimilation of hip-hop and EDM aesthetics and practices. EDM festivals had mainly been a European phenomenon, but in the 2000s, the US experienced a surge in the number of highly successful EDM pop festivals. ${ }^{5}$ This spawned a new generation of DJs that became super-stars, and the sound began to move into the mainstream as this new type of DJ-Producer became massively sought after in the pop music industry's "song machine" (Seabrook 2012). The skills, technology and aesthetics of DJing was thus imported to the pop charts, adding the role of the DJ-Producer to Paul Thebergés' definition of the "hyphenated musician", which already includes a combination of a singer-songwriterproducer-engineer-musician-sound designer (Théberge 1997: 221-22).

To track and exemplify the assimilation of hip-hop, EDM and digital temporalities, I will analyse three tracks, all from 2017, that represent differing temporalities as well as technology significations. 
INTERrelational Algorithms: "Scared to Be LOnely" (2017)

Garrix and Dua Lipa's hit "Scared to Be Lonely" is an example of the pop format's adaptation of EDM's "break routine", or what has been labelled the "pop drop" (Sloan and Harding, 2020: 46-52). ${ }^{6}$ From one perspective, this form represents an almost extreme teleological push with its overt and relentless revelling in setting up formal difference, announced extensively by framing devices. From another (macro) perspective, the break routine's formal units in the shorter pop format produce an element of formal circularity exceeding the traditional verse-prechorus-chorus formula. The same could be said of "Scared to Be Lonely" where one begins to predict the formal units' circular unfolding. Because of the formulaic order, the constant push towards the next formal unit and its dramatization, everything is expected. In other words, the track ambiguously exhibits both highly goaloriented forward-pushing linearity and formal circularity.

If we turn to the rhythmic elements of "Scared to Be Lonely", we hear that the climactic drop-like dance chorus is defined by a wall of bass and synthesizers. These all display the same envelope (understood as the way the sound changes over time). They do this in a very unusual manner defined by the algorithmic nature of compression. ${ }^{7}$ Compression follows an essential rule: if a signal's volume exceeds threshold $\mathrm{X}$, then attenuate the signal by the ratio Y. Sidechain compression is slightly more complicated and contains more directly interrelated processes across signals. It is basically the process of one sound's transients triggering a compressor that controls (and ducks the signal of ) one or several other signals. ${ }^{8}$ In EDM, this is often done so that a four-on-the-floor kick drum activates a compressor that ducks the volume of bass and synths. The result is that the wall of sound (and thus most of the track) bounces back up on the offbeat.

In "Scared to Be Lonely" the wall bounces to a different rhythm. It is difficult to aurally disentangle exactly how the interrelational dynamic processes are set up, but it is likely either an LFO-tool, a volume modulation plugin, or one or several sidechains with one of them being a so-called ghost sidechain trigger. ${ }^{9}$ The latter is a sound trigger that is not sent to the main output, so it is not heard, but it still works as a trigger for a sidechain compressor. In "Scared to Be Lonely", the ghost sidechain triggers on the 8ths, but with one or several beats lifted or missing resulting in the synth/bass-layer's uneven bounces. This irregular feel is amplified by the envelope settings' fairly slow attack and decay creating particularly slow swells and ducks which adds to the irregularity and fundamentally unnatural feel of the wall's collective envelope.

Regardless of the exact production methods utilised, the wall is essentially created and shaped by a chain of algorithms. The manner of its process (shaped through settings of factors such as ratio, envelope, knee-type, etc.) is part and parcel of its creative use. While various types of analogue compression have been used extensively by producers throughout recent electronic music history, the digital domain of contemporary DAW's afford new levels of control, automation, precision and intersectionality. From a groove perspective, the gridded drums and the wall's unusual envelope interconnect in a very particular way. 
While perhaps not qualifying as a groove from a traditional perspective, the wall does, however, display very tangible discrepancies. The gridded drums supply a background that the wall both bounces to and differs from in character and envelope. The discrepancies are repeated verbatim according to the exactness of the digital grid.

Recent developments in hip-hop do display some substantially different characteristics that I will now discuss with an example of trap hip-hop in pop music.

\section{Suspended Difference: "Rockstar (feAt. 21 SAVAGe)" (2017)}

Post Malone's "rockstar" excels in excessive sameness. Except for the introduction of the rapper 21 Savage and minor variations, the arrangement, dynamics and mood of the track stay the same for its almost four minutes long duration. It revels in its restrained lack of variation, drive or push. For instance, more than half of the track consists of the same twobar melody. The lyrics do change, but they are sung with an unusual lack of variation which is amplified by the heavy use of Auto-Tune. The beat is a typical trap beat with rolling hi-hat figures, the metallic "young chop snare" (from the producer Young Chop's sample library), and a boomy 808 kick-drum and a bass (which is probably a pitched kick-drum with a long decay).

The timing and timbral differences due to the minute velocity variations that traditionally define drum performance are not only quantised and repeated with exact sameness. They are also repeated in such a way that the reverb-less dry punches of the hi-hat and snare sound like on-off switches, punch-card coded in the grid of the piano-roll. Taking the practice of sequencing beats into account, where minute squares on the grid activate sounds, it is perhaps not surprising that the aesthetic outcome begins to resemble miniature auditory monoliths. Devoid of performative, corporeal or tactile gesture, it is the sound and signification of the sequencer and its operationality. While FL Studio is undoubtedly used for a plethora of musical styles that do not fit this interpretation, it is difficult not relate the sound of, for instance, trap music and the practices of FL Studio's digital sequencing. Here, instead of imagined narratives of performed, physical, gestural activity, the beat is embedded and defined by automation and digital multiplicity at the expense of human activity and agency.

Except for the intro and outro, the beat loops throughout the track. It breaks occasionally in a way that resembles a DJ turning the music down before a chorus or drop, yet here it is mostly followed by a return to the same continuous lead melody. The minor formal change in the module where the melody changes slightly and goes up is repeated twice by Post Malone leaving it somewhere between a chorus, bridge and prechorus. In this A', the rhythm in the melody stays more or less the same as in A. In other words, instead of a versechorus form, this is mainly one idea, one sound, one beat. The verse becomes the chorus and vice versa, and any sign of goal, direction, build-up or variation are punctured by the loop-based sameness. ${ }^{10}$

Instead of the golden-section aesthetics of a verse-chorus form's bridge, the introduction of 21 Savage happens exactly in the middle of the track (1:49 into the 3:38 long track). The 
palindromic form is not unusual in hip-hop and arguably adds to the overall repetitive nature. It is a principle of addition and division, blocks that fit and connect, loops that stack horizontally and vertically, order that defies formal tension. Indeed, time still passes, but kinaesthesia (understood as the sensation of moving) is deliberately deferred. It does not create a sense of time-pushing or striving for something else, and it is not based on irregularity or a stable instability. The suspended difference in "rockstar" aesthetically excels in and celebrates stable exactness with a lack of cuts, discrepancies, tension and teleological aspirations.

I will now turn to a pop track that is based on blending the production practices of both EDM and hip-hop.

\section{Groove as Technology-signification: "Chained to the Rhythm" (2017)}

Katy Perry's "Chained to the Rhythm" subscribes to a more conventional form and teleology. It begins with an intro of two bars. Here, the rhythm section of drums, bass and other indistinct instruments (mainly piano and keyboard) are bandpass-filtered so that only midfrequencies remain, resembling (a sample of) an old record. When the first verse begins, the song's main groove is established. It primarily consists of synthetic claps, bass guitar and the indistinct group of instruments from the intro, but the group is now panned wide.

In fact, this indistinct group of sound sources are grouped together in numerous unusual ways that all define the track's groove. While the claps are clear and quantised, the indistinct group and its shared rhythmic pattern is submitted to a collective envelope bouncing to a sidechain-compressor. The $95 \mathrm{bpm}$ sway of the song's groove is built on the sidechained group's collective envelope (again, it is difficult to decipher the programmed processes, but it sounds as if the sidechain trigger is either the kick drum, a ghost trigger or the bass). Besides the sidechain, a bandpass filter (different than the one in the intro) in the midrange frequencies adds to the group's sampled feel.

This is further amplified in the verse after the first chorus. Here, the claps are muted, and the indistinct group is hardly audible. But the frequencies are slowly swept back to include the upper registers due to two separate low-pass filters that gradually open up the threshold so that the respective upper frequencies of the sounds are let through. This creates movement and tension towards the pre-chorus.

In other words, the groove of the track is based on signifying the hip-hop practice of sampling breakbeats as wells as EQ-filtering and sweeps of EDM DJ'ing (just as the title is a reference to several classic house music tracks). While the former represents a circular temporality, the latter creates tension and linear variation, pushing time forward. This combination produces not only a temporal ambiguity, but also juxtaposes significations of genre-based practices that define the track's groove. The groove is thus based less on individual timing of sound sources and more on a highly mediatized group of instruments lacking individuality. Composing groove is thus less about moving individual sounds to an earlier or later position on a time axis as it is about the composite directly interrelated 
result across sound sources. It is more about setting up processes that shape collective inflections-or a lack thereof-in ongoing genre-signifying, interconnected negotiations.

\section{Perspectives for Pop as Process}

From a listening perspective, the production of pop music is increasingly characterised by a black-boxing of origins. The lack of distinguishable performance-based sound sources decreases the layman pop listener's chance to decipher the origins or the intricate technical processes that constitute a track. In the following, I will discuss how pop music's processuality can be interpreted as an intersection of a broad range of practices that entail a shift to a process paradigm. I will then compare this to the tracks analysed through the lens of new media theory.

In his analysis of interface-based music production, Mike D'Errico suggests the term "procedural listening". "[It is] a dominant skill developed by audio producers that allows both musicians and audiences to focus on the process-oriented mechanics of media forms, rather than simply audio content." (D'Errico 2016: 77, italics in original). Bridging the producer and the recipient, it connects with the listener orientation of the DJ or laptop-performer, where the processual character of the work installs a position of being both performer and observer. D'Errico argues how this entails a shift in the focus and the creativity towards the formal mechanics of the computational system (D’Errico 2016: 96).

It is essentially a position and an approach that stems from hip-hop and EDM's intertwined histories and aesthetics. The preference for loops and process-control rests upon both the creative (ab)use of technology and an Afrodiasporic adoration for the negotiation of the ongoing. For instance, Joseph G. Schloss describes how the aesthetics of sample-based hiphop is based on a veneration for processual mastery, where it is less about the significance of the individual sound objects and more about producer's skill in manipulating them (Schloss 2004: 159). It is a combination of cultural and aesthetic sensibilities that are met, hosted and expanded upon in particular usages of technology.

This has interpretive consequences for notions of groove, time and how they relate. In his analysis of the interaction between humans, data and nonhumans, sociologist Mike Michael describes what he calls co(a)gents. "The value of particular co(a)gents rests not so much on their empirical 'accuracy' as on their capacity to illuminate otherwise hidden processes" (Michael 2004: 10). Essentially, characteristics such as timing carry less meaning than the processual assemblage they help constitute. What's important is the way that details repeat. Correspondingly, groove has little to do with the discrepancy of, for instance, a snare drum, and more to do with what technological process the discrepancy signifies as part of an interrelational material whole. What does this type of looping signify?

The timing characteristics of the three songs analysed above all signify fairly concrete technological practices that again signify genre. In other words, in a step away from humanistic interpretations of music production, groove in contemporary pop music is increasingly produced by a particular entanglement between timbre and media materiality as they are defined by digital processes. 


\section{Prehensions in the GRID}

The grid rules in all the tracks analysed above. Arguably, it is most present in the sequenced trap-beat of "rockstar", but it also works as very active but to some extent silent partner in "Scared to Be Lonely" and "Chained to the Rhythm". Groove is thus created in a push and pull between quantised rigidity where sounds obey the grid directly and sounds that both connect and divert according to the grid indirectly. In other words, the sounds and the virtual reference structure are connected in differing ways. In this type of grid-based music, where the grid structure can literally grasp the sounds, we need to account for the reference structure and how it directly or indirectly affects the music's temporal features. The griddeviations of the wall in "Scared to Be Lonely" and the sample-like group in "Chained to the Rhythm" do seem paradoxical. From a performance-based perspective, they hold substantial discrepancies. Yet these are shaped in intricate, detailed, complicated and distinctly inhuman ways. The levels of complexity are humanly impossible, and these are then repeated verbatim as the unruly and somewhat unpredictable is ambiguously repeated with digital accuracy.

This processual intersection of human on non-human agencies begins to echo the process philosophy of Alfred North Whitehead. His fundamental concept that asks "how an actual entity becomes constitutes what that actual entity is" does indeed seem to resonate with contemporary culture (Whitehead 1978 [1929]: 23, italics in original). ${ }^{11}$ A central tenet in his process philosophy is the concept of prehensions that are "concrete facts of relatedness" (Whitehead 1978:23). Luciana Parisi relates Whiteheads process ontology to digital media and how they are defined by prehensions that grasp, connect, include and exclude, and transform data, thereby defining what an entity is and how it relates to others (Parisi 2013: XII). Adding this perspective to the interpretation of digital pop music's processes puts the emphasis not on static entities, but the mutually constitutive character of shaping sound. How are individual sounds shaped by combinations of functions?

For instance, the staging of Dua Lipa's vocal is defined by not only reverb, but reverb combined with several functions including various types of dynamic compression and gating. It is also a process of grasping, including, excluding and transforming. These operations entail agencies of both subjects (Lipa and the producer) and objects (the algorithmic processes of the DAW's signal processing). Interpreting the wall of "Scared to Be Lonely" is essentially an analysis of how the grid grasps the functions of one or several sidechain compressors that control the envelope of multiple sounds. Interpreting pop music thus becomes an analysis of how entities specifically relate across microcosm and macrocosm. Arguably, "rockstar" demonstrates less direct interrelations across sound sources, but it still revels in the multiplicity of functions across the particular and the general.

New essential questions arise: what is the relationship between individual sounds and the grouping of them and how are they grouped? With, for example, MIDI, many seemingly different sources-pitch-control information (e.g., Auto-Tune or Melodyne), dynamic control (such as compression) or frequency filters - can become similar or even 
one (like the grouping of sounds in "Scared to Be Lonely" and "Chained to the Rhythm"). Conversely, one source can become many when the same metadata is applied to multiple sources. Grouping sounds is not a new thing. But connecting, interrelating and multiplying in the DAW is not just easier; it is part and parcel of the compositional practice. When sounds increasingly interrelate or are subject to master effects, it blurs distinctions and creates a de-hierarchisation of individual sounds and metadata. This turn to the assemblages of prehensions therefore involves a shift of focus towards how data (audio or metadata) multiply across a track, how (often algorithmic) processes interrelate and which patterns they form.

\section{Topology, Temporal Ambiguity and the Human Scale}

The three tracks are all fundamentally based on setting up, controlling, editing and adjusting running processes. Even so, the outcomes are very different. In "Scared to Be Lonely", the overtly teleological pop drop dynamic seemingly poses a stark contrast to the suspended difference of the trap circularity in "rockstar". Lury, Parisi and Terranova argue how contemporary society is increasingly subject to a topological rationality. This "participates in and renews the specificity of the material and the sensuous", and is "dynamic, soft and tractable, both precise and vague, able to operate the physical and sensual horizon of experience beyond and beneath the law-like symbolic system of signification" (Lury, Parisi and Terranova 2012: 28). It is a logic in which figures, forms and patterns emerge and disappear and it entails a loss of fixity, distance and perspective. As such, "Scared to Be Lonely" can be heard as a reflection of this topological flux. Yet, "rockstar" seemingly defies this. Music history is ripe with examples of static or circular music, but "rockstar"'s innately digital character gains a new level of precision, multiplicity and fundamentally a different value coming from the inside of the digital's topological nature.

From this perspective, the trap track begins to exemplify what media theorist Wolfgang Ernst calls the true luxury of today's temporal economy: the resistance towards permanent change. In other words, the excessive difference-defying sameness afforded by digital music production and exhibited in "rockstar" not only reflects a societal shift towards precision and synchronisation. It is at the same time an aesthetic opposition to contemporary society's topological character. As music as well as an algorithmic structure, the excessive negation of difference in "rockstar" bends and negates its own topological nature.

The substantial differences and embedded temporal ambiguities of the three tracks seem to echo similar ambiguities in new media networks' temporalities. Indeed, time is ambiguous in ubicomp culture. On one hand, media have become the social metronomes of the everyday as our lives are increasingly defined by digital exactness (Neverla 2010: 183). Instantaneity, coordination and synchronization rule as the local constantly links to the global. Digital media plant temporal flags with exact precision and divide time with infinitesimal accuracy. Yet, on the other hand, information is also constantly permutating. Persistence meets flux 
in the ambiguous duality between the relentless presence and a constant development of cloud-based computing. Concurrently, our access to the past and its juxtaposition with the present is changed by the expansion of information. In contemporary society we work polychronically; we engage in multiple activities simultaneously and place less value on temporal order. As Couldry and Hepp argue, temporality becomes heterogenous (Couldry and Hepp 2016: 107). The issue is not so much a lack of time to react, but a lack of time for interpretation or making narrative sense (Couldry and Hepp 2016: 114).

From WolfgangErnst's media archaeological perspective, sound is liberated from semantics and hermeneutics as "the microphysical close reading of sound, where the materiality of the recording medium itself becomes poetical, dissolves any semantically meaningful archival unit into discrete blocks of signals" (2012: 60, italics in original). As a consequence, " $[\mathrm{m}]$ edia culture turns from phonocentrism to mathematics, liberating sonicity from the conventional time domain" (Ernst 2016: 118). ${ }^{12}$ Ernst's media materialism defies direct reference, but still holds signifying capabilities beyond direct conscious attention. This does not mean that Ernst rejects social, political or economic factors, but he proposes a media materialism that accounts for a lack of human gesture.

Digital processes flatten the hierarchy and level the ground between the human and its coexistence with the nonhuman. The implications for pop music seem quite important. For one, a programming paradigm at the expense of a recorded performance paradigm involves two very different types of mediatization. One is based on utilizing hardware and treating recorded sound. The other is based on processual metadata-control and cloudbased databases. One rests upon a medial transformation, whereas the other entails the media itself as an environment; this is again part of networked computational culture. One is based on human agency, and the other substantially utilizes distributed agency that includes digital automation to a degree where loops and, more generally, processes form interrelational algorithmic structures. Agency is innately entangled with, divided amongst and dispersed to actors outside the human realm, and this challenges the pedestal of the human subject and its perception. ${ }^{13}$

This inherent controlling of processes puts the producer's agency in a place where performance, control and programming meet. It relies on digital algorithms' abilities to predict dynamic fluctuations and allows for precision and control to an unprecedented degree. New levels and kinds of complexity are created in the infinitesimal details that are subject to discrete variations between loops and interrelational functions across sound sources. This demands new kinds of attention, skill and talent from the producer. The craft or creativity consists of setting, editing and adjusting interrelational processes through combinations of mainly metadata. Yet, this control is also met with a level of stochasticism embedded in the interrelated algorithmic agency. In the hybridization of human and machinic agency, the art lies in controlling or even taming the automated processes and how they combine. 


\section{TACIT KNOWLEDGE}

From a listening perspective, this means that temporality manifests differently. The (Kantian) claim that phenomena depend upon the mind to exist is thereby suspended. This arguably presents some challenges for phenomenological understandings of digitalization. Mark Hansen has, in a long line of publications, critiqued overtly phenomenological interpretations of twenty-first century media. He argues that ubicomp culture essentially disjoins worldly temporalization from human temporalization due to the sheer speed and infinitesimal detail that can be adjusted and repeated beneath the threshold of human attention. It is essentially a question of scale and complexity. "Ubicomp environments, precisely because they offer information peripherally-which is also to say, in a timeframe beneath the threshold of conscious perception-catalyze sensation according to a protocol that is not wholly biotic, but is crucially and irreducibly technical" (Hansen 2013: 72).

To Hansen, ubiquitous computing makes the microtemporal sensuous. Arguably, few art forms revel as much in sensuous microtemporality as digital music. Pop's digital temporality, its scale, its hybridized agency, its level of complexity including the staging of the microtemporal challenges overt anthropocentric interpretations. Like the inherent, immanent character of ubicomp that exists in the peripheral background, beyond or outside human perception, so do the processes that constitute digital pop.

However, instead of completely writing intentionality off in a black-boxing of algorithmic processes, Taina Bucher argues that "tacit knowledge" of the underlying logic of algorithmic systems linger in background (2018: 62). ${ }^{14}$ The same argument could be made for listening to digital processes. It is a processual unfolding that manifests less in relation to direct or conscious interpretations. In this shift from perception to sensation, musical referentiality gains an indirect character where it is more subject to prereflexive experience of the logic of the music unfolding.

The processual transferral of inscriptional value also offers a somewhat different approach to technological evocations of the past. It adds a temporal element to the opaqueness of mediation. However, it is more akin to Wolfgang Ernst's beforementioned prereflexive media archaeology than notions of sonic patina that nostalgically transport the listener to days gone by or retromania. ${ }^{15}$ The palette of technology-embedded temporalities that manifest as signifiers of practices and genre are experienced preconsciously.

Similarly, process philosophy's disbandment of the subject as primary or originary also entails a particular mode of registering data where participation precedes recognition. This is not to say that listening to contemporary pop music is devoid of consciousness perception. But, compared to performed music, pop music in the twenty-first century represents a different, digital process-based ontology that suggests different aesthetic potentialities and lends itself to novel kinds of listening. This is less about interpretation, semantics, phenomenological intentionality or teleology-inducing difference. It is tacit knowledge lingering in the background of the manner in which musical processes unfold. 
A strict process paradigm will deny repetition without difference. However, combining process ontology with phenomenological listening arguably presents some fundamental problems, as the latter is based on a unified conscious perception. Process ontology will deny the privileging of a unified conscious perception because of the multitude of objectsubject independent temporal unfoldings. The increased privileging of technological agency challenges the human performance logic of groove theory as well as musicological interpretations of time in general. It represents a departure from correlationism and phenomenological interpretations of musical time. Instead, topology and heterogenous temporality signify new process-bound interpretations of musical time.

It puts the emphasis on how music unfolds in time qualitatively. Digital pop music offers very intricate and particular technology-signifying heterogenous temporalities that are based on a negotiation of the relationship between music and time in ways that are innately digital. To resign the temporalities of digital pop to an overt unilinear flux that does not distinguish between the hows, the manner, the types of unfolding in its distributed agencies, would be a disservice. Because it is exactly here-in the ambiguity and complexity of the temporal — that the listening pleasure of digitally produced pop music resides.

\section{NOTES}

1 I discuss the new generation of DAWs in further detail in the article "Who Let the DAWs Out? The Digital in a New Generation of the Digital Audio Workstation" (Reuter, forthcoming in Popular Music and Society).

2 Danielsen's "Presence and Pleasure: The Funk Grooves of James Brown and Parliament" (2006) has been highly influential in groove studies, and her research later continued into digital music (see, e.g. 2010, 2018, 2019, 2020; Brøvig-Hanssen and Danielsen 2016).

3 See also Danielsen (2006: 162). Danielsen distinguishes between what she calls "even rhythm on the grid, on the one hand, and deep, groovy rhythmic designs, on the other" (Danielsen 2019: 596). Scepticism towards exact, non-different repetition is fairly common in groove studies. Luis-Manuel Garcia argues that exact repetition has been the subject of a devaluation where it is put in a position of "the Other" compared to repetition with a difference (Garcia 2005: 5). When Russell Potter distinguishes between what he calls blank repetition and repetition with a difference, Garcia duly criticizes Potter for claiming that a certain acceptable level of repetition elevates jazz, blues and rap at the expense of the more quantized grooves of disco, techno and other dance music genres (2005: 5).

4 See Théberge (1997), Möllenkamp (2014) and Strachan (2017).

5 For more on the history of EDM pop, see Reynolds (2012), Matos (2015), Holt (2017) and Gálvez (2019). Mark Butler argues that EDM has "emerged as a label for recent, commercially popular genres such as dubstep" (Butler 2014: $5 \mathrm{fn}$. 4). He emphasizes that popular as well as academic writing since the 1990s has generally used the term to encompass all genres within the category of electronic dance music and that this definition should not be confused with the narrower, more recent one. To separate the general label EDM from the more 
recent iteration, I use the label EDM pop, as Holt (2017) and James (2015) do. It defines mainly house and dubstep-inspired, drop-based electronic music that in a recorded music context abides to a pop single length, format and function. I am acutely aware of the almost blasphemic potential here. For instance, Mandy-Suzanne Wong writes that many authors feel that EDM outside clubs and raves is an aesthetical failure (2013: 195). However, my purpose and focus here is not a comparison, but to track changes in pop.

6 Traditional EDM DJ sets last hours and a break routine usually lasts between twenty minutes and multiple hours, so even though this form is always moving towards the drop, parts may very well be experienced as stand-stills. However, the timeframe changes when the break routine is imported to the three or four-minute musical format of a pop track. Because there is significantly less time between the breakdown and the drop, EDM pop tracks often display intense momentum-building and contrasts between breakdown, build up and the drop. EDM pop can also display very intricate formal recombinations that blur distinctions between pre-chorus, chorus, post-chorus and the pop drop. For more on the mere resent iterations of popular music form, see Barna (2020) or Peres (2018).

7 Cormen et al. offers a definition of an algorithm. "Informally, an algorithm is any well-defined computational procedure that takes some value, or set of values, as input and produces some value, or set of values, as output. An algorithm is thus a sequence of computational steps that transform the input into the output" (Cormen et al. 2009: 13).

8 See Hodgson (2011) and D'Errico (2015).

9 It has not been possible to settle the exact technique used here. However, the point is more the algorithmic character entailed in contemporary production methods. For example, plugins such WavesFactory's Trackspacer, Cableguys' VolumeShaper or Polyverse's Gatekeeper offer intricate volume modulation control that expand the accessibility and possibilities of traditional use of lateral dynamic processing (such as the compressor, gate, limiter, expander).

10 The main variation is the addition of 21 Savage. His vocal is significantly different, with less reverb, lower frequencies and more compression, which is a vocal staging with substantially more proximity than Post Malone's reverberated voice. 21 Savage's lower frequency range compliments the track's overall frequency response, and its drier, closer sound offers a sense of relief from Post Malone's distance. Furthermore, 21 Savage initially abides by the lead melody, but begins to break out and rap or sing more freely, seemingly almost improvisational, which is intensified when the track switches briefly to the A' before returning to the lead melody with Post Malone as the lead vocalist. In other words, the introduction of 21 Savage can be heard as a sort of a bridge or solo, and it offers a shift or release in the track's excessive sameness.

11 New media researchers have, in various ways, employed the process-relational philosophy of Alfred North Whitehead to account for computational culture and contemporary society in general (e.g. Shaviro, 2009; Hansen, 2015; Bucher, 2018). For instance, Steve Shaviro writes, "in a world where everything from music to DNA is continually being sampled and recombined, and where the shelf life of an idea, no less than of fashion in clothing, can be measured in months if not weeks, Whitehead's question is the truly urgent one" (2009: X). Luciana Parisi has in a long line of publications engaged with Whitehead to account for the aesthetics of algorithmic structures in relation to how they reveal the limits of rationality and perception (see Parisi 2013, 2015, 2016, 2017a, 2017b). 
12 Ernst's media materialism is heavily influenced by a German media studies and particularly Friedrich Kittler (and to some extent also Foucault's reading of media). Kittler's influential writings traced the invention of the alphabet to the distinct emergence of analogue media (such as the gramophone) that again lead to digitalization and the computer (see, for example, Kittler 1999). For Kittler, the move is from the symbolic order to one that essentially rules out human perception and thus also hermeneutic sense-orientation as well as phenomenological strategies (Kittler 2017).

13 In his analysis and comparison of early minimalism and disco, Robert Fink makes a likeminded argument. He writes how the hour-long repetitions of minimalism and (ecstasyfuelled) weekend-raves challenge the human scale of classical musical teleology, which is based on goal-direction and "a basic phenomenological congruence with the way we perceive quotidian bodily rhythms" (Fink 2005: 44). Due to the ambiguous nature of repetition, he suggests the non-binary term "recombinant teleology" (Fink 2005: 42-7).

14 The term originally stems from Michael Polanyi, see Polanyi (1966).

15 See Katz (2010: 155) and Reynolds (2011).

\section{REFERENCES}

Agawu, Kofi. 1995. "The Invention of 'African Rhythm”. Journal of the American Musicological Society 48(3): 177-92. <https://doi.org/10.2307/3519832>.

Barna, Alyssa. 2020. "The Dance Chorus in Recent Top-40 Music". The Society for Music Theory Videocast Journal 6(4). <http://doi.org/10.30535/smtv.6.4 >.

Brøvig-Hanssen, Ragnhild and Anne Danielsen. 2016. Digital Signatures: The Impact of Digitization on Popular Music Sound. Cambridge: MIT Press. <https://doi.org/10.7551/mitpress/10192.001.0001>.

Bucher, Taina. 2018. If...Then: Algorithmic Power and Politics. New York: Oxford University Press.

Butler, Mark. J. 2006. Unlocking the Groove: Rhythm, Meter, and Musical Design in Electronic Dance Music. Indianapolis: Indiana University Press.

- - 2014. Playing with Something That Runs: Technology, Improvisation, and Composition in DJ and Laptop Performance. New York: Oxford University Press. < https://doi.org/10.1093/acprof:oso/9780195393613.001.0001>.

Cormen, Thomas H., Charles E. Leiserson, Ronald L. Rivest and Clifford Stein. 2009. Introduction to Algorithms. 3rd Ed. MIT Press.

Couldry, Nick and Andreas Hepp. 2016. The Mediated Construction of Reality. Oxford: Polity Press.

D’Errico, Mike. 2015. “Off the Grid: Instrumental Hip-Hop and Experimentalism after the Golden Age”. In The Cambridge Companion to Hip-Hop, ed. Justin A. Williams, 280-91. Cambridge: Cambridge University Press. <https://doi.org/10.1017/cco9781139775298.026>.

_- _. 2016. "Interface Aesthetics: Sound, Software, and the Ecology of Digital Audio Production”. Ph.D. dissertation, UCLA.

Danielsen, Anne. 2006. Presence and Pleasure: The Funk Grooves of James Brown and Parliament. Middletown, Wesleyan University Press. 
- - . 2010. "Introduction: Rhythm in the Age of Digital Reproduction". In Musical Rhythm in the Age of Digital Reproduction, ed. Anne Danielsen, 1-16. Farnham: Routledge.

- _- 2018. "Time and Time Again: Repetition and Difference in Repetitive Music". In Over and Over: Exploring Repetition in Popular Music, ed. Olivier Julien and Christophe Levaux, 37-50. New York: Bloomsbury Academic.

- _ . 2019. "Glitched and Warped: Transformations of Rhythm in the Age of the Digital Audio Workstation". In The Oxford Handbook of Sound and Imagination: Volume 2, ed. Mark Grimshaw-Aagaard, Mads Walther-Hansen and Martin Knakkergaard, 593-609. Berlin: Springer. <https://doi.org/10.1093/oxfordhb/9780190460242.013.27>.

- - - 2020. "Desktop Production and Groove". In The Bloomsbury Handbook of Music

Production, ed. Simon Zagorski-Thomas and Andrew Bourbon, 267-280. New York: Bloomsbury Academic \& Professional. < https://doi.org/10.5040/9781501334054.ch-018>.

Ernst, Wolfgang. 2012. "Media Archaeography: Method and Machine versus the History and Narrative of Media". In Digital Memory and the Archive, ed. Wolfgang Ernst and Jussi Parikka, 55-73. Minneapolis: University of Minnesota Press. <https://doi.org/10.5749/minnesota/9780816677665.003.0004 >.

- - . 2016. Sonic Time Machines, Explicit Sound, Sirenic Voices, and Implicit Sonicity. Amsterdam: Amsterdam University Press.

- - 2018. "Tracing Tempor(e)Alities in the Age of Media Spatio-Temporal Media Analytics". Media Theory 2(1): 164-80.

Fink, Robert. 2005. Repeating Ourselves: American Minimal Music as Cultural Practice. Berkeley: University of California Press.

_- - 2011. "Goal-Directed Soul? Analyzing Rhythmic Teleology in African American Popular Music". Journal of the American Musicological Society 64(1): 179-238. $<$ https://doi.org/10.1525/jams.2011.64.1.179>.

Gálvez, José. 2019. "On Analyzing EDM DJ Sets: Problems and Perspectives for a Sociology of Sound”. In Contemporary Popular Music Studies, ed. Marija Dumnić Vilotijević and Ivana Medić, 149-59. Wiesbaden: Springer Fachmedien. < https://doi.org/10.1007/978-3-658-25253-3 14>.

Garcia, Luis-Manuel. 2005. "On and On: Repetition as Process and Pleasure in Electronic Dance Music”. Music Theory Online 11(4): 1-14. < https://mtosmt.org/issues/mto.05.11.4/ mto.05.11.4.garcia.pdf $>$, (accessed 15 November 2021).

Goodman, Steve. 2008. “Timeline (Sonic)”. In Software Studies: A Lexicon, ed. Matthew Fuller, 256-59. Cambridge, MA: MIT Press.

Hansen, Mark. 2009. "Living (with) Technical Time: From Media Surrogacy to Distributed Cognition”. Theory, Culture and Society 26(2-3): 294-315.

_- _. 2012. "Technics Beyond the Temporal Object”. New Formations 77: 44-62. $<$ https://doi.org/10.3898/newf.77.03.2012>.

- _- 2013. "Ubiquitous Sensation or The Autonomy of the Peripheral: Towards an Atmospheric, Impersonal, and Microtemporal Media". In Throughout: Art and Culture Emerging With Ubiquitous Computing, ed. Ulrik Ekman, 63-88. Cambridge, MA: MIT Press. - - 2015. Feed-Forward: On the Future of Twenty-First-Century Media. Chicago: University of Chicago Press. 
- - 2016. "Topology of Sensibility”. In Ubiquitous Computing, Complexity, and Culture, ed. Ulrik Ekman, Jay David Bolter, Lily Diaz, Morten Sondergaard and Maria Engberg, 33-47. New York/London: Routledge.

Hodgson, Jay. 2011. "Lateral Dynamics Processing in Experimental Hip Hop: Flying Lotus, Madlib, Oh No, J-Dilla and Prefuse 73". Journal of the Art of Record Production 5. $<$ https://www.arpjournal.com/asarpwp/lateral-dynamics-processing-in-experimental-hiphop-flying-lotus-madlib-oh-no-j-dilla-and-prefuse-73/>, (accessed 15 November 2021).

Holt, Fabian. 2017. "EDM Pop: A Soft Shell Formation in a New Festival Economy”. In Weekend Societies, ed. Graham St. John, 25-43. New York: Bloomsbury Publishing Inc. <https://doi.org/10.5040/9781501311413.ch-002>.

James, Robin. 2015. Resilience \& Melancholy: Pop Music, Feminism, Neoliberalism. Ropley: John Hunt Publishing.

Katz, Mark. 2010. Capturing Sound: How Technology Has Changed Music (Rev. Ed.). Berkeley: University of California Press.

Keil, Charles. 1966. "Motion and Feeling through Music". The Journal of Aesthetics and Art Criticism 24(3): 337-49. <https://doi.org/10.1111/1540 6245.jaac24.3.0337>.

- - - 1987. "Participatory Discrepancies and the Power of Music". Cultural Anthropology 2(3): 275-83. <https://doi.org/10.1525/can.1987.2.3.02a00010>.

Keil, Charles and Steven Feld. 1994. Music Grooves: Essays and Dialogues. Chicago: University of Chicago Press.

Kittler, Friedrich. 1993. Draculas Vermächtnis - Technische Schriften. Leipzig: Reclam Verlag.

-_-. 1999. Gramophone, Film, Typewriter. Stanford: Stanford University Press.

_- _. 2017. "Real Time Analysis, Time Axis Manipulation”. Cultural Politics 13(1): 1-18. $<$ https://doi.org/10.1215/17432197-3755144>.

Kramer, Jonathan. D. 1988. The Time of Music: New Meanings, New Temporalities, New Listening Strategies. New York: Schirmer.

Langer, Susanne. K. 1953. Feeling and Form: A Theory of Art. New York: Charles Scribner's Sons.

Lury, Celia, Luciana Parisi and Tiziana Terranova. 2012. "Introduction: The Becoming Topological of Culture”. Theory, Culture \& Society 29(5): 3-35. <https://doi.org/10.1177/0263276412454552>.

Michael, Mike. 2004. “On Making Data Social: Heterogeneity in Sociological Practice”. Qualitative Research 4(1): 5-23. < https://doi.org/10.1177/1468794104041105>.

Matos, Michaelangelo. 2015. The Underground Is Massive: How Electronic Dance Music Conquered America. New York: Dey Street Books.

Möllenkamp, Andreas. 2014. "Paradigms of Music Software Development". National Institute for Music Research, Berlin: 9th Conference on Interdisciplinary Musicology. <https:// www.kulturwissenschaften.uni-hamburg.de/personen/moellenkamp-2014-paradigms.pdf $>$, (Accessed 8 November 2021).

Parisi, Luciana. 2013. Contagious Architecture: Computation, Aesthetics, and Space. Cambridge, MA: MIT Press.

- _ . 2015. "Cutting Away from Smooth Space”. In The Lure of Whitehead, ed. Nicholas Gaskill and A. J. Nocek, 267-96. Minneapolis: University of Minnesota Press. $<$ https://doi.org/10.5749/minnesota/9780816679959.003.0011 >. 
- - - 2016. "Automated Thinking and the Limits of Reason". Cultural Studies $<->$ Critical Methodologies 16(5): 471-81. <https://doi.org/10.1177/1532708616655765>.

_- _. 2017a. "After Nature: The Dynamic Automation of Technical Objects". In Posthumous Life: Theorizing Beyond the Posthuman, ed. Jami Weinstein and Claire Colebrook, 155-78. New York: Columbia University Press. <https://doi.org/10.7312/wein17214-008>.

- _ . 2017b. "Computational Logic and Ecological Rationality". In General Ecology: The New Ecological Paradigm, ed. Erich Hörl and James Burton, 75-100. London: Bloomsbury Publishing Plc. <https://doi.org/10.5040/9781350014725.ch-002>.

Peres, Asaf. 2018. "Everything You Need to Know About the Postchorus". Top40 Theory. $<$ https://www.top40theory.com/blog/everything-you-need-to-know-about-the-postchorus $>$, (accessed 3 June 2020).

Polanyi, Michael. 1966. The Tacit Dimension. Garden City: Doubleday \& Company, Inc.

Potter, Russell. A. 1998. "Not the Same: Race, Repetition, and Difference in Hip-Hop and Dance Music". In Mapping the Beat: Popular Music and Contemporary Theory, ed. Thomas Swiss, Andrew Herman and John Sloop, 31-46. Malden, MA: Blackwell Malden.

Reynolds, Simon. 2011. Retromania: Pop Culture's Addiction to Its Own Past. London: Faber \& Faber.

- - . 2012. "How Rave Music Conquered America". The Guardian. <http://www.theguardian. $\mathrm{com} / \mathrm{music} / 2012 / \mathrm{aug} / 02 /$ how-rave-music-conquered-america $>$, (accessed 6 February 2020).

Rietveld, Hillegonda. C. 2018. "Machine Possession - Dancing to Repetitive Beats". In Over and Over: Exploring Repetition in Popular Music, ed. Olivier Julien and Christophe Levaux, 60-67. New York: Bloomsbury Academic. <https://doi.org/10.5040/9781501324871.ch-005>.

Schloss, Joseph G. 2004. Making Beats: The Art of Sample-Based Hip-Hop. Middletown: Wesleyan University Press.

- - - 2014. Making Beats: The Art of Sample-Based Hip-Hop, 2nd Ed. Middletown: Wesleyan University Press.

Seabrook, John. 2012. “The Song Machine”. The New Yorker 88(6): 48-55. March 19.

Shaviro, Steven. 2009. Without Criteria: Kant, Whitehead, Deleuze, and Aesthetics. Cambridge, MA: MIT Press.

Sloan, Nate, and Charlie Harding. 2020. Switched on Pop: How Popular Music Works, and Why It Matters. New York: Oxford University Press.

Snead, James A. 1984. "Repetition as a Figure of Black Culture”. In Black Literature and Literary Theory, ed. Henry Louis Gates Jr., 59-79. London/New York: Methuen.

Solberg, Ragnhild Torvanger. 2014. “'Waiting for the Bass to Drop': Correlations Between Intense Emotional Experiences and Production Techniques in Build-up and Drop Sections of Electronic Dance Music". Dancecult 6(1): 61-82. $<$ https://doi.org/10.12801/1947-5403.2014.06.01.04 >.

Strachan, Robert. 2017. Sonic Technologies: Popular Music, Digital Culture and the Creative Process. New York: Bloomsbury Academic \& Professional.

Théberge, Paul. 1997. Any Sound You Can Imagine, Making Music / Consuming Technology. Hanover, N.H/London: Wesleyan University Press.

Whitehead, Alfred North. 1978. Process and Reality. New York: The Free Press. 
DISCOGRAPHY

Katy Perry. 2017. Chained to the Rhythm. Witness. Capitol. <https://www.discogs.com/master/1193114-Katy-Perry-Witness >

Martin Garrix \& Dua Lipa. 2017. Scared to Be Lonely. Stmpd Rcrds/Epic. <https://www.discogs.com/release/9742067-Martin-Garrix-Dua-Lipa-Scared-To-Be-Lonely> Post Malone. 2017. rockstar (feat. 21 Savage). Republic Records. <https://www.discogs.com/release/11104981-Post-Malone-Feat-21-Savage-Rockstar> 